Check for updates

Cite this: Phys. Chem. Chem. Phys., 2019, 21, 24296

Received 25th July 2019, Accepted 24th September 2019

DOI: $10.1039 / c 9 c p 04156 \mathrm{~g}$

rsc.li/pccp

\section{Dendronised Ni(II) porphyrins as photoswitchable contrast agents for MRI $\dagger$}

\author{
Marcel Dommaschk, (D) a Jens Gröbner, ${ }^{a}$ Vanessa Wellm, (D) a \\ Jan-Bernd Hövener, (D) ${ }^{b}$ Christian Riedel $^{c}$ and Rainer Herges (D) *a
}

Light-responsive contrast agents for magnetic resonance imaging (MRI) based on $\mathrm{Ni}(\mathrm{II})$ porphyrin molecular spin switches have recently been introduced. We present their implementation in water and methanol based gels leading to the first soft materials that are rewritable with light and readable with MRI. Light of two different wavelengths as non-invasive stimuli can be applied to switch MRI contrast on and off, with a high spatiotemporal resolution and without fatigue.

Magnetic resonance imaging (MRI) is a non-invasive 3D imaging modality with unsurpassed soft-matter contrast. Paramagnetic contrast agents (e.g. gadolinium complexes) are used to enhance structural contrast in tissues and materials. ${ }^{1}$ Opposed to structural imaging is functional MRI with "smart" contrast agents that report on various parameters, such as $\mathrm{pH}$, temperature or parameters

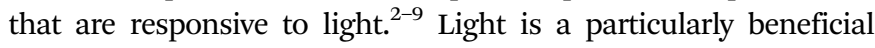
stimulus, because it can be applied non-invasively with high spatiotemporal resolution, during MRI scans. ${ }^{10,11}$ Contrast switching with light in soft or heterogeneous materials and in living tissues opens a number of applications in chemical engineering and medicine, such as in situ studies of processes during catalytic reactions, ${ }^{12,13}$ and the characterisation of mass transport in heterogeneous polymers or elastomers ${ }^{13}$ including the monitoring of controlled drug delivery from polymer gels. ${ }^{14,15}$ We now report on the synthesis and investigation of molecular spin switches in aqueous solution (including human serum), as well as in methanol and water based gels. We investigate the properties of these materials towards applications in medical and material imaging. The synthesis of the light-responsive but non-water-soluble Ni(II) porphyrins 1 and 2 is established and their switching properties are investigated. ${ }^{16-18}$

${ }^{a}$ Otto-Diels-Institute of Organic Chemistry, Christian Albrechts University Kiel, Otto Hahn Platz 4, 24118 Kiel, Germany. E-mail: rherges@oc.uni-kiel.de

${ }^{b}$ Section Biomedical Imaging, MOIN CC, Kiel University, Am Botanischen Garten 14, 24118 Kiel, Germany

${ }^{c}$ Clinic for Radiology and Neuroradiology, University Medical Center SchleswigHolstein, Arnold Heller Str. 3, 24105 Kiel, Germany

$\dagger$ Electronic supplementary information (ESI) available. See DOI: 10.1039/c9cp04156g 1st and 2nd generation glycerol dendrimers (H-G[1.0] and H-G[2.0], Fig. 1) are well known to improve water solubility even of highly hydrophobic compounds and were prepared as described previously. ${ }^{19-22}$ The experimental procedures for the introduction of the dendrimers are provided in Section SI of the ESI. $\dagger$ The protected dendronised Ni(II) porphyrins 1-G[1.1], 1-G[2.1], 2-G[1.1] and 2-G[2.1] are obtained in yields $>70 \%$. The subsequent quantitative acetal deprotection yields the dendronised Ni(II) porphyrins 1-G[1.0], 1-G[2.0], 2-G[1.0] and 2-G[2.0] (Fig. 1).

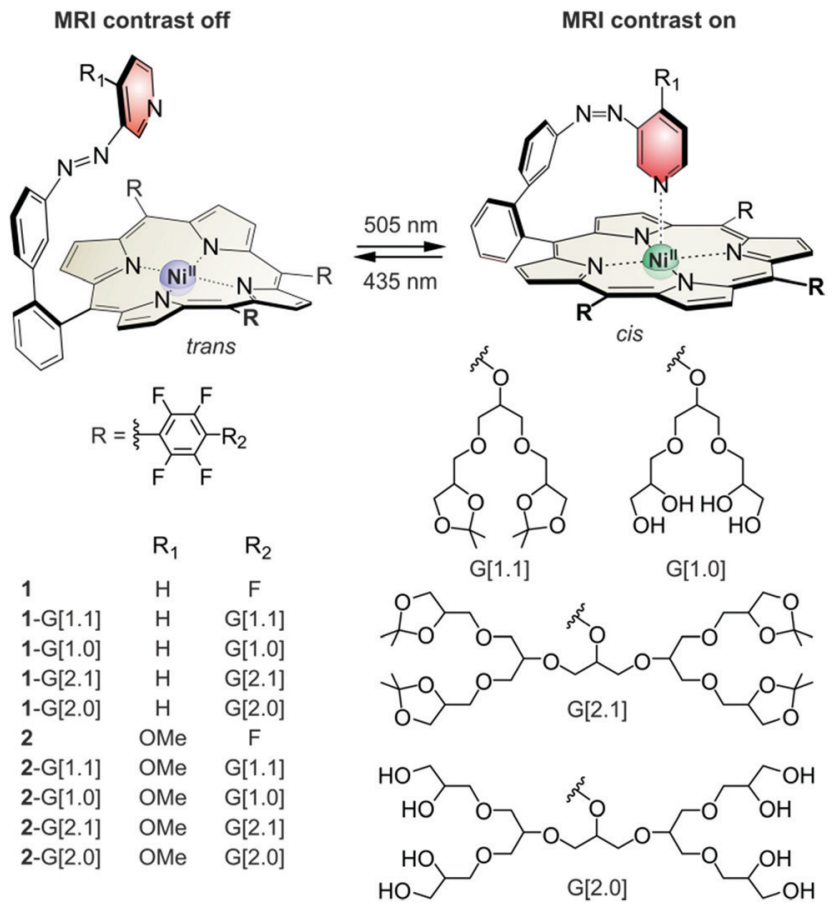

Fig. 1 Dendronised porphyrins 1-G[1.1], 1-G[1.0], 1-G[2.1], 1-G[2.0], 2-G[1.1], 2-G[1.0], 2-G[2.1] and 2-G[2.0] were obtained by funtionalisation of 1 and 2 (Section $\mathrm{SI}, \mathrm{ESI} \dagger$ ). All molecules undergo light-driven coordination-induced spin state switching (LD-CISSS) between the paramagnetic 5-coordinate state and the diamagnetic 4-coordinate state using green (495-530 nm) and blue light $(420-435 \mathrm{~nm})$ respectively. 
Although 1-G[1.0] and 2-G[1.0] already have 12 hydroxy groups, they are not water soluble at all. The 1st generation glycerol dendrimer is apparently too small and the hydrophobic character of the $\mathrm{Ni}$ (II) porphyrin dominates the molecular property. In contrast, the solubility characteristics of the 2nd generation dendronised Ni(II) porphyrins 1-G[2.0] and 2-G[2.0] are dominated by the hydrophilic dendrimer. The Ni(II) porphyrins exhibit an unlimited miscibility with water and protic solvents such as methanol. The 2nd generation dendrimer efficiently prevents the aggregation of the hydrophobic porphyrin cores leading to high solubility. Further evidence for the non-aggregation is the absence of intermolecular coordination. The non-dendronised Ni(II) porphyrins 1 and 2 exhibit dimer formation (intermolecular coordination) and thus, become paramagnetic with increasing concentration. ${ }^{17}$ This process is unwanted because it reduces the efficiency of contrast switching. The dimer formation can be investigated via ${ }^{1} \mathrm{H}-\mathrm{NMR}$ spectroscopy. The experiment was performed in DMSO- $\mathrm{d}_{6}$ as it is the only mutual solvent for 2 and 2-G[2.0]. An increasing concentration of 2 gives rise to massive signal broadening and shifting (Fig. 2, left). The bulky 2nd generation dendrimers prevent the formation of dimers and the accompanying paramagnetism. The ${ }^{1} \mathrm{H}-\mathrm{NMR}$ signals of $2-\mathrm{G}[2.0]$ are virtually concentration-independent (Fig. 2, right). The remarkable solubility characteristics obtained by dendronisation are a useful tool for porphyrin chemistry in general with a broad variety of applications.

All presented Ni(II) porphyrins undergo light-driven coordinationinduced spin state switching (LD-CISSS). The isomerisation of the azopyridine changes the coordination number and thereby the spin state of nickel. The thermodynamic stable trans isomer cannot coordinate intramolecularly. With a coordination number of four, the Ni(II) porphyrin is diamagnetic and MRI silent (MRI contrast off). The cis isomer coordinates intramolecularly. With a coordination number of five, the Ni(II) porphyrin is paramagnetic and MRI active (MRI contrast on). The two isomers interconvert upon irradiation with green light (495-530 nm, trans to cis) and blue light (420-435 nm, cis to trans). As presented previously, the switching efficiency (difference between the minimum and maximum amount of the isomers obtained by irradiation) of the $\mathrm{Ni}(\mathrm{II})$ porphyrins strongly depends on the intramolecular coordination strength. ${ }^{16}$ While the cis to trans isomerisation with blue light is reliably quantitative ( $>95 \%$ ), the trans to $\mathrm{cis}$ isomerisation with green light is facilitated by a strong intramolecular coordination.

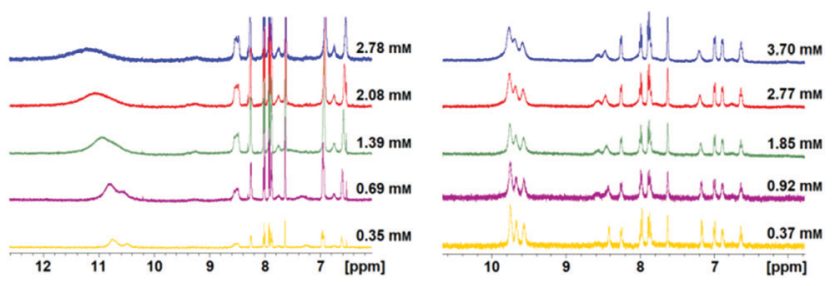

Fig. 2 Partial ${ }^{1} \mathrm{H}-\mathrm{NMR}$ spectra (DMSO- $\mathrm{d}_{6}, 300 \mathrm{~K}$ ) of 2 (left) and 2-G[2.0] (right) at different concentrations. The signal broadening and shifting in the case of $\mathbf{2}$ indicate increasing paramagnetism due to intermolecular coordination. Dendronised 2-G[2.0] does not show a similar effect even at higher concentrations.
$\mathrm{Ni}(\mathrm{II})$ porphyrin 1 with pyridine as an axial ligand exhibits a photostationary state (PSS) of $65 \%$ cis isomer (495 nm, acetone). ${ }^{17}$ $\mathrm{Ni}(\mathrm{II})$ porphyrin 2 with the stronger binding 4-methoxy pyridine exhibits a PSS of $>95 \%$ cis isomer under the same conditions. ${ }^{16,23}$ Functionalisation with the glycerol dendrimers affects the binding constant and thus influences the switching properties. The binding constant is lowered because the electron withdrawing para fluorine atoms are substituted by the electron donating glycerol dendrimer. This affects the PSS of the derivatives of 1 . The influence of the acetal deprotection is minor and the generation of the dendrimer has no influence at all. Thus, the switching efficiency has the order of $1>1-G[1.1]=1-G[2.1]>1-G[1.0]=1-G[2.0]$ (Fig. S1 left, ESI $\dagger$ ). This trend is not observed for the derivatives of $\mathbf{2}$ where PSS is largely independent of the meso-substituent (switching efficiency: $2=2-G[1.1]=2-G[2.1]=2-G[1.0]=1-G[2.0]$, Fig. S1 right, ESI $\dagger)$. Apparently, the stronger binding of the para-methoxy pyridine overcompensates the loss of binding affinity caused by the dendrimers providing an almost quantitative photoswitching of all five derivatives in organic solvents. However, switching MRI contrasts of an aqueous solution is much more challenging. The donor strength of pyridine ligands is drastically reduced due to hydrogen bonding with water. Disappointingly, the switching efficiency of 1-G[2.0] drops to almost zero when dissolved in water. The most likely reason for this finding is that pyridine is too weak a ligand and cannot coordinate to the Ni(II) porphyrin in the presence of water. In contrast, 2-G[2.0] bearing the stronger binding methoxy pyridine ligand retains a dia- to paramagnetic switching efficiency of 19\% (Section SII.4, ESI $\dagger$ ). This efficiency is sufficient to perform MRI contrast switching in water (Fig. S13, ESI $\dagger$ ) and even in human serum (Fig. 3). 2-G[2.0] is the first molecule performing LD-CISSS in aqueous solution and the first MRI contrast agent that changes between a MRI silent and a MRI active state upon light irradiation. ${ }^{10}$ The thermal half-life of cis $2-\mathrm{G}[2.0]$ in water at body temperature $\left(37^{\circ} \mathrm{C}\right)$ is 39 days which is more than sufficient for MRI applications (Section SII.5, ESI $\dagger$ ).

The relaxivity in water of $2-\mathrm{G}[2.0]$ in its contrast-on-state is $0.03 \mathrm{~s}^{-1} \mathrm{mM}^{-1}$ (Section SIII.3, ESI $\dagger$ ). The poor performance is due to the decreased switching efficiency in water, which allows a maximum concentration of $19 \%$ paramagnetic species (Section SII.4, $\mathrm{ESI} \dagger$ ). The relaxivity of the contrast-on-state in methanol is much better $\left(0.191 \mathrm{~s}^{-1} \mathrm{mM}^{-1}\right.$, Section SIII.3, ESI $\left.\dagger\right)$ and similar to previously presented non-dendronised Ni(II) porphyrins. ${ }^{16}$ Improvement of the performance in water is the next step towards the application of the photoswitchable Ni(II) porphyrins and can be realised with even stronger ligands such as imidazole. ${ }^{24,25}$

The remarkable solubility characteristics of the dendronised $\mathrm{Ni}(\mathrm{II})$ porphyrins allow the immobilisation in gels to investigate the spatial resolution of the light-induced MRI contrast switching. Previous attempts using $\mathbf{1}$ and $\mathbf{2}$ failed due to agglomeration and poor solubility. The dendronised Ni(II) porphyrins 2-G[2.1] and 2-G[2.0] were incorporated with two different gels: (a) a methanol based gel containing the gelator $( \pm$ )-trans-1,2-bis(dodecylureido)cyclohexane $^{26,27}$ and (b) an agar hydrogel. UV-vis spectroscopy proves that the switching efficiency is not hampered by the gels. LD-CISSS is as efficient as in solution (Fig. S2, ESI $\dagger$ ). Diffusion of the large molecules is slow and doesn't affect the measurements. 

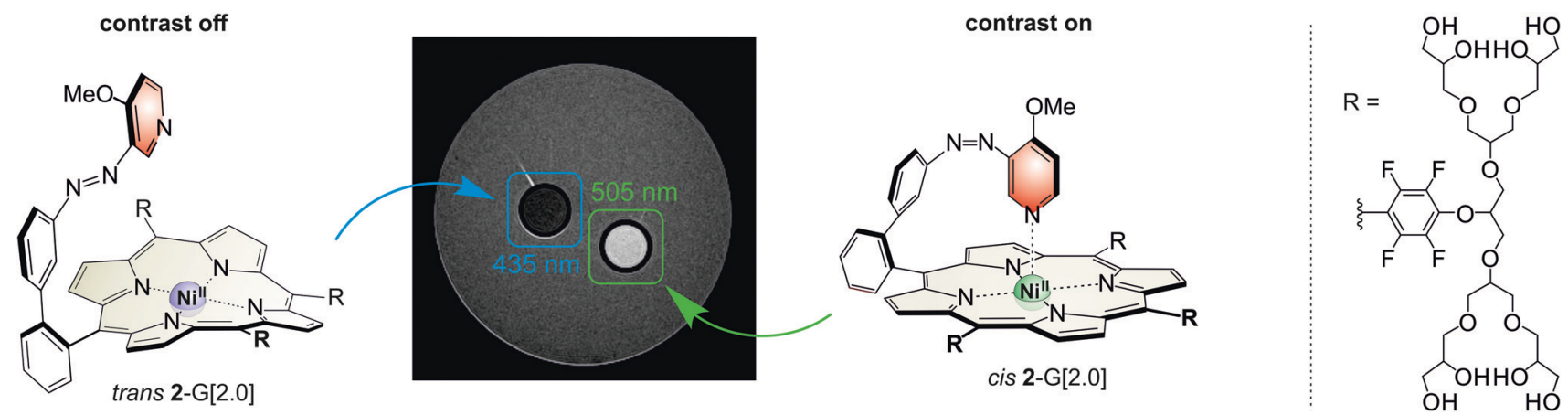

Fig. 3 MR image of $5 \mathrm{mM} 2-\mathrm{G}[2.0$ ] solution in human serum irradiated with blue (435 nm, left) and green light ( $505 \mathrm{~nm}$, right) respectively. The solutions are kept in NMR tubes in an agar phantom. Experimental details are provided in Section SIII.2 of the ESI. $\dagger$

a)

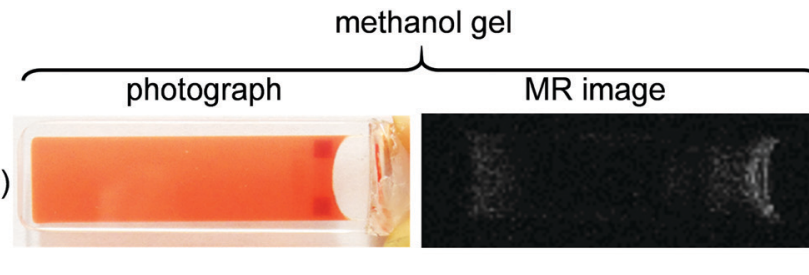

b)

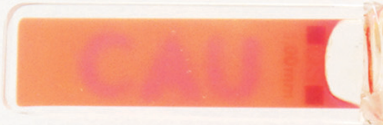

c)

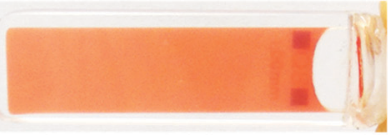

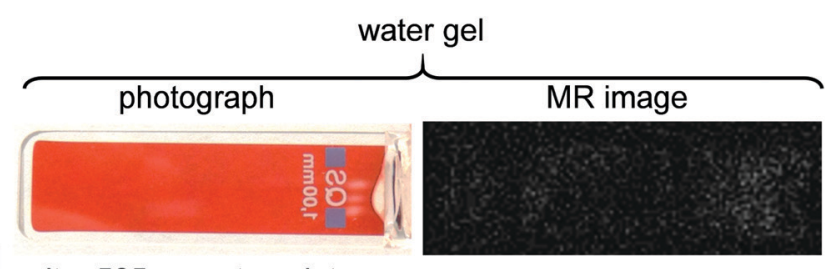

write: $505 \mathrm{~nm}+$ template
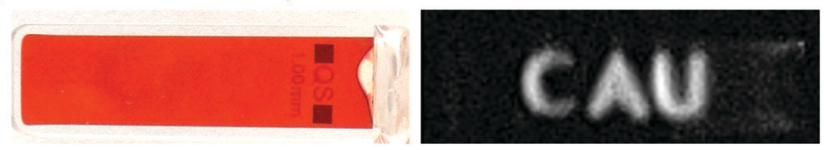

delete: $435 \mathrm{~nm}$
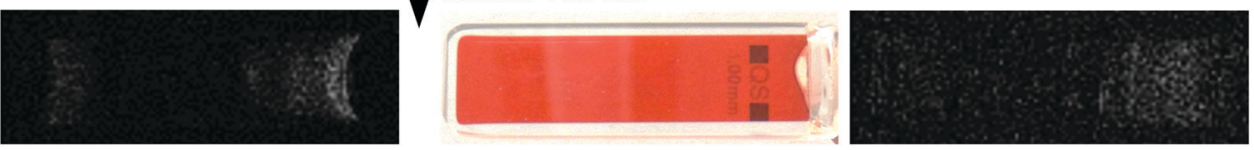

Fig. 4 Photographs and MR images of gels in cuvettes $(30 \times 10 \times 1 \mathrm{~mm})$ containing the switchable contrast agents. Left: Methanol gel containing Ni(॥) porphyrin 2-G[2.1] (0.8 mM), right: water based gel (agar) containing Ni porphyrin 2-G[2.0] (5.0 mM). (a) Before irradiation, (b) after irradiation with green light $(505 \mathrm{~nm})$, (c) after irradiation with violet light $(435 \mathrm{~nm})$. The nominal resolution of the letters CAU (Christian Albrechts University) amounts to $0.33 \times$ $0.33 \times 1 \mathrm{~mm}$. Experimental details are provided in Section SIII. 2 of the ESI.†

To investigate the spatial resolution, we prepared a $0.8 \mathrm{mM}$ solution of $2-\mathrm{G}[2.1]$ in the methanol gel. The gels were prepared in cuvettes of the dimension $3.0 \times 1.0 \times 0.1 \mathrm{~cm}$. The cuvette was irradiated with green light $(505 \mathrm{~nm})$ for 1 min using a template of letters CAU. Conversion to the paramagnetic cis isomer is barely visible with the unaided eye (Fig. $4 \mathrm{~b}$, left). The MR-image of the gel, however, clearly reveals the pattern (Fig. 4b). The gel can be erased by irradiation with blue light and the sample can be rewritten. Equivalent to the parent systems $\mathbf{1}$ and 2, the dendronised Ni(II) porphyrins show no sign of fatigue over many cycles (Section SII.3, ESI; $\dagger>100000$ cycles performed for 2 previously). ${ }^{16}$ The effect using $2-\mathrm{G}[2.0]$ in a hydrogel (agar) is similar but slightly less distinct. Due to the lower switching efficiency a higher concentration ( $5 \mathrm{mM}$ instead of $0.8 \mathrm{mM}$ ) is required to obtain a similar contrast difference. The higher concentration demands a longer irradiation time of $10 \mathrm{~min}$ (in $\mathrm{MeOH}$ gel: $1 \mathrm{~min}$ ). To the best of our knowledge the 2-G[2.1] and 2-G[2.0] gels are the first light-writable and MRI-readable materials. The writing can be clearly recognised by MRI proving that we can address and read the contrast agent with a submillimetre resolution.
Dendronised molecular spin switches based on photoswitchable Ni(II) porphyrins were used to realise MRI contrast on-off switching in aqueous solution and in human serum. No sign of fatigue was observed after repeated switching cycles. Both states are stable for weeks (thermal half-life of the on-state is 39 days at body temperature). The incorporation of methanol and water based gels does not impede the switching efficiency with respect to the pure solvents. The gels are the first lightwriteable (and rewriteable) MRI-readable materials. With a light addressable spatial resolution of $\sim 200 \mu \mathrm{m}$, a number of unprecedented applications in medical imaging, interventional radiology and imaging of mass transport in soft and heterogeneous materials arises. Further studies are devoted to improving the switching efficiency and relaxivity by the replacement of the pyridine ligand by imidazole, ${ }^{24}$ or by replacing $\mathrm{Ni}^{2+}$ with $\mathrm{Fe}^{3+}$ within the porphyrin core. ${ }^{28,29}$

\section{Conflicts of interest}

There are no conflicts to declare. 


\section{Acknowledgements}

The authors gratefully acknowledge the funding from the Deutsche Forschungsgemeinschaft for the Collaborative Research Centre "SFB 677 Function by Switching”. JBH acknowledges support by the DFG (HO 4604/2-2, GRK 2154/1-2019, HO 4604/3-1, PMI 1267). Kiel University and the Medical Faculty are acknowledged for supporting the Molecular Imaging North Competence Center (MOIN CC) as a core facility for imaging in vivo. MOIN CC was founded by a grant from the European Regional Development Fund (ERDF) and the Zukunftsprogramm Wirtschaft of SchleswigHolstein (Project no. 122-09-053).

\section{Notes and references}

1 P. Hermann, J. Kotek, V. Kubíček and I. Lukeš, Dalton Trans., 2008, 3027-3047.

2 C. Tu and A. Y. Louie, Chem. Commun., 2007, 1331-1333.

3 C. Tu, E. A. Osborne and A. Y. Louie, Tetrahedron, 2009, 65, 1241-1246.

4 L. M. De Leon-Rodriguez, A. J. M. Lubag, C. R. Malloy, G. V. Martinez, R. J. Gillies and A. D. Sherry, Acc. Chem. Res., 2009, 42, 948-957.

5 É. Tóth, R. D. Bolskar, A. Borel, G. González, L. Helm, A. E. Merbach, B. Sitharaman and L. J. Wilson, J. Am. Chem. Soc., 2005, 127, 799-805.

6 M. Woods, G. E. Kiefer, S. Bott, A. Castillo-Muzquiz, C. Eshelbrenner, L. Michaudet, K. McMillan, S. D. K. Mudigunda, D. Ogrin, G. Tircsó, S. Zhang, P. Zhao and A. D. Sherry, J. Am. Chem. Soc., 2004, 126, 9248-9256.

7 S. Zhang, K. Wu and A. D. Sherry, Angew. Chem., 1999, 111, 3382-3384.

8 S. Aime, S. G. Crich, M. Botta, G. Giovenzana, G. Palmisano and M. Sisti, Chem. Commun., 1999, 1577-1578.

9 J. Hall, R. Häner, S. Aime, M. Botta, S. Faulkner, D. Parker and A. S. de Sousa, New J. Chem., 1998, 22, 627-631.

10 K. Kruttwig, D. R. Yankelevich, C. Brueggemann, C. Tu, N. L'Etoile, A. Knoesen and A. Y. Louie, Molecules, 2012, 17, 6605-6624.

11 E. A. Osborne, B. R. Jarrett, C. Tu and A. Y. Louie, J. Am. Chem. Soc., 2010, 132, 5934-5935.

12 L. F. Gladden, Top. Catal., 1999, 8, 87-95.
13 A. K. Whittaker, eMagRes, 2012, 1, 103-108, DOI: 10.1002/ $9780470034590 . e m r s t m 1269$.

14 J. H. P. Collins, L. F. Gladden, I. J. Hardy and M. D. Mantle, Appl. Magn. Reson., 2007, 32, 185-204.

15 C. M. Terry, L. Li, H. Li, I. Zhuplatov, D. K. Blumenthal, S.-E. Kim, S. C. Owen, E. G. Kholmovski, K. D. Fowers, R. Rathi and A. K. Cheung, J. Controlled Release, 2012, 160, 459-467.

16 M. Dommaschk, M. Peters, F. Gutzeit, C. Schütt, C. Näther, F. D. Sönnichsen, S. Tiwari, C. Riedel, S. Boretius and R. Herges, J. Am. Chem. Soc., 2015, 137, 7552-7555.

17 M. Dommaschk, C. Schütt, S. Venkataramani, U. Jana, C. Näther, F. D. Sönnichsen and R. Herges, Dalton Trans., 2014, 43, 17395-17405.

18 S. Venkataramani, U. Jana, M. Dommaschk, F. D. Sönnichsen, F. Tuczek and R. Herges, Science, 2011, 331, 445-448.

19 M. Dommaschk, F. Gutzeit, S. Boretius, R. Haag and R. Herges, Chem. Commun., 2014, 50, 12476-12478.

20 C. Kördel, C. S. Popeney and R. Haag, Chem. Commun., 2011, 47, 6584-6586.

21 M. Wyszogrodzka and R. Haag, Chem. - Eur. J., 2008, 14, 9202-9214.

22 M. Wyszogrodzka, K. Möws, S. Kamlage, J. Wodzińska, B. Plietker and R. Haag, Eur. J. Org. Chem., 2008, 53-63.

23 F. Gutzeit, M. Dommaschk, N. Levin, A. Buchholz, E. Schaub, W. Plass, C. Näther and R. Herges, Inorg. Chem., 2019, DOI: 10.1021/acs.inorgchem.9b00348.

24 G. Heitmann, C. Schütt, J. Gröbner, L. Huber and R. Herges, Dalton Trans., 2016, 45, 11407-11412.

25 G. Heitmann, C. Schütt and R. Herges, Eur. J. Org. Chem., 2016, 3817-3823.

26 J. H. van Esch, F. Schoonbeek, M. de Loos, H. Kooijman, A. L. Spek, R. M. Kellogg and B. L. Feringa, Chem. - Eur. J., 1999, 5, 937-950.

27 K. Hanabusa, K. Shimura, K. Hirose, M. Kimura and H. Shirai, Chem. Lett., 1996, 885-886.

28 M. K. Peters, S. Hamer, T. Jäkel, F. Röhricht, F. D. Sönnichsen, C. von Essen, M. Lahtinen, C. Naether, K. Rissanen and R. Herges, Inorg. Chem., 2019, 58, 5265-5272.

29 S. Shankar, M. Peters, K. Steinborn, B. Krahwinkel, F. D. Sönnichsen, D. Grote, W. Sander, T. Lohmiller, O. Rüdiger and R. Herges, Nat. Commun., 2018, 9, 4750. 